\title{
EARLY QUASI-STEADY ELECTRO-MAGNETIC FIELDS ABOUT CONDUCTING SURFACES
}

\author{
GRAHAM J. WEIR ${ }^{1}$
}

(Received 24 April 1998)

\begin{abstract}
Step changes in current through either grounded or ungrounded wires lying on the surface of a uniformly conducting half-space produce image current sources within the surface of the conductor. This image current is effectively the only source term for initial changes in $\partial_{1} B_{z}, E_{x}$ and $E_{y}$. The general steady state electric and magnetic field components resulting from steady currents flowing through either grounded or ungrounded wires of finite length lying on the surface of a uniform half- space are derived. Then the operators mapping these steady fields into the early values of $\partial_{t} B_{z}, E_{x}$ and $E_{y}$ on or above the conducting half-plane resulting from instantaneously stopping the current flow through the wires are derived.
\end{abstract}

\section{Introduction}

Geophysical prospecting is used widely in the exploration of mineral and geothermal resources. Electrical currents are forced to flow through the earth, generating measurable electric fields at the surface. Mineral resources and hot saline water from geothermal fields produce anomalous regions of conductivity within the earth, and so can be detected from anomalies in the corresponding surface values of the electromagnetic field.

Perhaps the most widely used method in geophysical prospecting utilises the Wenner array. Steady currents flow through different lengths of wire, thus probing the earth to different depths. The resulting steady electrical potentials are measured by grounded wires approximately parallel to the current-carrying wires.

Another widely used method employs a current-loop. Transient vertical magnetic fields can be measured by such loops, either by altering the current source in the loop, and observing the resulted induced fields in the same loop, or in a nearby loop. From

\footnotetext{
${ }_{1}^{1}$ Applied Mathematics Group, The New Zealand Institute for Industrial Research and Development, P O Box 31-310, Lower Hutt, New Zealand.

(C) Australian Mathematical Society 2001, Serial-fee code 0334-2700/01
} 
the methods of measurement widely used today, the three most important observables are $\partial_{t} B_{z}$ (horizontal ungrounded loops), $E_{x}$ and $E_{y}$ (horizontal grounded wires). Here $\partial_{t}$ is the partial derivative with respect to time $t ; x$ and $y$ are horizontal Cartesian coordinates; $z$ is a vertically upward coordinate; $B_{z}$ is the vertical component of the magnetic field; and $E_{x}, E_{y}$ are the horizontal components of the electric field. Recent instrumentation will allow other components to also be measured. This paper will pay special attention to the observables $\partial_{t} B_{z}, E_{x}$ and $E_{y}$.

Measuring systems often record data which is not analysed. For example, the transient fields generated by establishing or removing the steady fields used in the Wenner array are not used (usually) in analyses. Significant advances in instrumentation [5] have provided more detail on the behaviour of these transient fields. The main aim of this paper is to provide an analysis of the fields which are generated soon after changes in current flow through either grounded or ungrounded insulated wires. In principle, this will provide complementary, but independent, parameters on the conducting properties of the earth, from those which are available using standard methods of analysis.

This paper considers initially steady current flows, and corresponding steady initial electromagnetic fields. At time $t=0$, these current flows are suddenly stopped, and eventually the associated electromagnetic fields decay to zero. Three phases [15] in this decay are recognised.

Firstly, no changes occur at any point on or above the surface until the speed of light travel time $\sqrt{\mu \epsilon} r$ has elapsed, where $r$ is a typical distance between the current source and observer. With the arrival of the speed of light signals, new fields are established on and above the surface within several $\epsilon / \sigma$ time units. The third phase is associated with the decay of the fields, as currents within the lower conducting half-plane diffuse away, with a time constant of $\mu \sigma r^{2}$. Within the conductor, displacement currents associated with the speed of light terms strongly damp changes, so that in practice only the third phase occurs within the conductor. Here induced polarisation effects are ignored; $\mu$ is the permeability of vacuum; $\epsilon$ permittivity of vacuum; and $\sigma$ the conductivity (for $z<0$ ).

For example, choosing the distance between the source and receiver $r$ as 10 kilometres and $\sigma^{-1}$ as $100 \mathrm{ohm}$-metres, gives the three time scales $\sqrt{\mu \epsilon} r, \epsilon / \sigma$ and $\mu \sigma r^{2}$ as $10^{-4}, 10^{-9}$ and 1 seconds, respectively. Most analyses are applied after the "one second" lapse, or within the third phase associated with diffusion of fields within the conductor. This paper aims to analyse the fields established after " $10^{-4}+10^{-9}$ " seconds, but before "1" second; that is, after the second phase, but before the third phase.

The analyses in this paper are a specialisation of general results [7] on electromagnetic propagation about conducting surfaces [2]. Sommerfeld used a Hertz vector formulation and obtained the general solution to a constant frequency dipole 
source about the surface. He showed that in addition to the source dipole, an image dipole about the surface and a continuous distribution of images are needed to satisfy Maxwell's equations. The corresponding solutions in the time domain [4] (rather than the frequency domain) were obtained by Wait $[10,11]$, who pointed out that the solutions for impulsive $(I(t)=I \delta(t))$ current sources follow directly from Sommerfeld's results. However, it was not possible in general to obtain through mathematical analysis the fields established by step changes in current. In general, numerical methods are needed to perform the convolutions connecting the solutions for step changes in current to the solutions from impulsive current sources. However, the corresponding solutions in a wholespace have been found [14].

This paper derives the general solutions on and above the conducting surface for $\partial_{t} B_{z}, E_{x}$ and $E_{y}$ resulting from step changes in current, for the time period after the arrival of the speed of light terms, but before the diffusive decay terms become important. In other words, new results are obtained, extending the work of Sommerfeld, but through the compromise of restricting the time period for which they are valid.

This paper divides naturally into two parts. The first part derives the steady state electric and magnetic fields resulting from wires of finite length, either grounded or ungrounded, on the surface of a uniform half-space. The second part of the paper derives the early quasi-steady state $\partial_{t} B_{z}, E_{x}$ and $E_{y}$ components, which are related to the initial steady field values, and so from the steady state results in the first part of the paper, the required general solution is obtained for the quasi-steady $\partial_{t} B_{z}, E_{x}$ and $E_{y}$ components.

\section{Steady state fields}

This section considers the steady state electric and magnetic fields generated by steady currents flowing through a system of grounded and ungrounded wires of finite length lying on the surface of a uniform half-space. Points where a wire is grounded are called electrodes, which are assumed to have zero spatial extent. Wires may overlay one another, or branch, or have common electrodes. The linearity of Maxwell's equations [8] suggests that wires, electrodes and currents can be superimposed, but Figure 1 shows that some constraint on such superpositions will be needed in general.

Cases A, B, C and D in Figure 1 represent the four possible permutations of currents of 1 and 2 amps entering a junction or node, and 3 amps leaving the node. Case $A$ has the three wires joined, and ungrounded. Case $B$ has the three wires grounded, and joined to a common electrode. Case $\mathrm{C}$ has one wire (carrying $1 \mathrm{amp}$ ) grounded to an electrode, and one wire with a discontinuous change in current. Case $\mathrm{D}$ is analogous to Case $C$. 


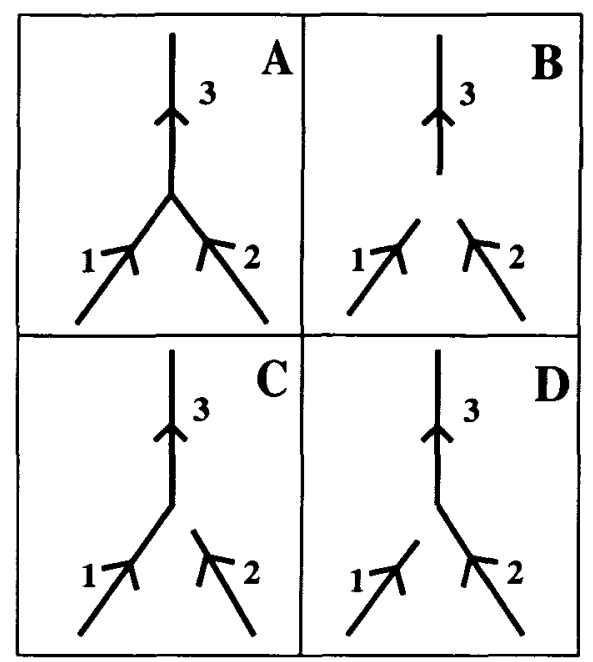

FIGURE 1. Example illustrating the need for constraints on the superposition of wires. Cases A (no electrodes) and $B$ (three coincident electrodes) are equivalent, but Cases $C$ and $D$ (one electrode) produce different fields from either $A$ or $B$.

Later in this section the steady electric and magnetic fields resulting from current flow in sections of wire, and due to the presence of electrodes, are derived. These solutions show that Cases A and B are equivalent, but are not equivalent to either' Cases $C$ or $D$, because the latter have net current flows into the earth of 1 and 2 amps respectively.

Consequently, Kirchhoff's Law of current conservation must be assumed for the surface wires, which implies that the net current to the earth from a system of wires is zero.

Consider one electrode connected to a semi-infinite vertical wire carrying current $I$ into the earth. From symmetry, the steady magnetic field associated with this current system [12] is axial,

$$
B_{\phi}= \begin{cases}\frac{-\mu I}{2 \pi r \sin \theta} & z>0 \quad(0 \leq \theta<\pi / 2), \\ -\frac{\mu I \cot (\theta / 2)}{2 \pi r} & z<0,\end{cases}
$$

where $(r, \theta, \phi)$ are spherical polar coordinates, centred on the electrode. This magnetic field satisfies

$$
\nabla \times B_{\phi}= \begin{cases}(0,0,0) & z>0 \\ \left(\frac{\mu I}{2 \pi r^{2}}, 0,0\right) & z<0\end{cases}
$$


which is consistent with a steady current $I$ flowing into an electrode, since $\nabla \times B=$ $\mu \sigma E$, and

$$
E=\left(\frac{I}{2 \pi \sigma r^{2}}, 0,0\right)
$$

Since tangential components of $E$ are continuous across the surface $z=0,(2.2)$ is also the required solution for the electric field above the surface.

From the Biot-Savart Law [3], the semi-infinite wire contributes an axial magnetic field

$$
b_{\phi}=\frac{-\mu I(1+z / r)}{4 \pi R^{2}}
$$

and subtracting (2.3) from (2.1) gives the effective axial magnetic field from an isolated electrode as

$$
B_{e \phi}=\frac{-\mu I(r-|z|)}{4 \pi r R}
$$

where $R^{2}=x^{2}+y^{2}$.

The contribution to the magnetic field from the wires is

$$
\begin{aligned}
B_{w} & =\nabla \times A_{w}^{0}, \\
\mathbf{A}_{w}^{0} & =\sum_{w} \frac{\mu I_{w}}{4 \pi} \int \frac{d \mathbf{l}_{w}}{r},
\end{aligned}
$$

where $r$ is the distance between the wire and the observer, $d \mathbf{l}_{w}$ is a vectorial integration along a wire in the direction of current flow, and the summation is over all wire segments, $w$. There is no electric field generated from the vector potential $\mathbf{A}_{w}^{0}$ in (2.5).

The general solution of the steady state electric field is therefore obtained by summing fields of the form (2.2) over each electrode position, and recalling that (2.2) is a radial field centred on an electrode position. Similarly, the general steady state magnetic field is obtained by summing the contributions in (2.4) from each electrode position, recalling that (2.4) describes an axial field centred on each electrode, and then adding the contributions from (2.5) for current flow through the wires.

In later sections it will be of critical importance to observe that the vector potential $\mathbf{A}_{w}^{0}$ in (2.5) has only horizontal components, and so the only contribution to the steady state vertical magnetic field results from current flow through the wires, since the contribution from (2.4) has no vertical component. Additionally, the vertical component of the steady magnetic field depends on the particular positioning of the wires, and not on the positions of the electrodes alone. 
To emphasise that the vector potential in (2.5) is dependent on wire positions, consider an infinitesimal small loop of wire of radius $R_{0}$, carrying current $I$ in an anticlockwise direction, with the centre at the origin of a Cartesian coordinate system. Then the vector potential, generated from (2.5), is a non-zero axial field at $(x, y, z)$,

$$
A_{w \phi}=\frac{\mu I R_{0}^{2}\left(x^{2}+y^{2}\right)^{1 / 2}}{4\left(x^{2}+y^{2}+z^{2}\right)^{3 / 2}}
$$

which would have to be zero, if (2.5) produced results independent of the positioning of the wires.

\section{Transient vertical magnetic field}

The aim of this section is to derive the early behaviour of the vertical component of the magnetic field which results from instantaneously stopping the current flows in the surface wires considered in the last section. The differential equation for $B_{z}$ is

$$
\nabla^{2} B_{z}=\mu \sigma \partial_{t} B_{z}+\mu \epsilon \partial_{t}^{2} B_{z}
$$

where $\nabla^{2}$ is the Laplacian, and $\partial_{t}$ differentiation with respect to time, and $\sigma, \epsilon$ are assumed constant with respect to frequency.

The "natural" non-dimensionalisation of (3.1) is obtained by scaling distance and time by respectively $r_{0}$ and $t_{0}$, where

$$
r_{0}=(1 / \sigma) \sqrt{\epsilon / \mu}, \quad t_{0}=\epsilon / \sigma
$$

which places the three terms in (3.1) on an equal footing. Choosing $\sigma^{-1}=100 \mathrm{ohm}$ metres fixes $r_{0} \sim 0.3$ metres and $t_{0} \sim 10^{-9}$ seconds, both of which are too small to be of physical importance in geophysical prospecting. Consequently, the time scale needed is not $t_{o}$, but $t_{0} \tau$, where $\tau$ is some large number. This is equivalent to setting $\epsilon$ to zero in (3.1), and requires the new time scale $t_{0} \tau$ to be $\mu \sigma L^{2}$, where $L$ is some typical length scale.

Later in this paper some of the mathematical expressions will be simplified by setting $\epsilon$ to zero, which is called the "quasi-steady" approximation. Although this approximation is justified through dimensional analysis, dimensional parameters will be retained throughout this paper, primarily because the general nature of the problem formulated here does not produce a natural length scale (other than $r_{0}$ ).

To proceed, double horizontal Fourier transforms of (3.1) are taken, yielding

$$
\mathscr{F} \nabla^{2} B_{z}=\partial_{z}^{2} \mathscr{F} B_{z}-\lambda^{2} \mathscr{F} B_{z} \text {, }
$$


where

$$
\begin{aligned}
k^{2} & =k_{x}^{2}+k_{y}^{2} \quad \text { and } \\
\mathscr{F} B_{z} & =\frac{1}{2 \pi} \int_{-\infty}^{\infty} d x \int_{-\infty}^{\infty} d y e^{i\left(k_{x} x+k_{y} y\right)} B_{z} .
\end{aligned}
$$

Consequently, the Fourier transform of any steady state field component (satisfying Laplace's equation) has the form

$$
\mathscr{F} B_{z}^{0}=\tilde{B}_{z}^{0} e^{-k|z|}
$$

where $\tilde{B}_{z}^{0}$ is independent of spatial position.

Taking the Laplace transform of (3.1),

$$
\mathscr{L} B_{z}=\int_{0}^{\infty} e^{-s t} B_{z} d t
$$

and the double horizontal Fourier transform, and using (3.3) yields

$$
\begin{aligned}
\partial_{z}^{2} \mathscr{F} L B_{z} & =\lambda^{2} \mathscr{F} L B_{z}-(\mu \sigma+\mu \epsilon s) \mathscr{F} B_{z}^{0}, \\
\lambda^{2} & =k^{2}+\mu \sigma s+\mu \epsilon s^{2},
\end{aligned}
$$

where $B_{z}^{0}$ is the initial value of $B_{z}$, and so

$$
\mathscr{F} L B_{z}=B^{ \pm} e^{-\lambda|z|}+\frac{\tilde{B}_{z}^{0}}{s} e^{-k|z|},
$$

where $B^{ \pm}$denote the two spatially independent coefficients above and below the conducting surface. Similarly, from (3.9), above and below the surface $z=0, \lambda$ has different values which are denoted by $\lambda^{+}, \lambda^{-}$respectively.

The coefficients $B^{ \pm}$are obtained from application of the boundary conditions on $B_{z}$; that is, that $B_{z}$ and $\partial_{z} B_{z}$ are continuous at $z=0$, and so from (3.10)

$$
\mathscr{F} L B_{z}=\frac{-2 \tilde{B}_{z}^{0} k}{s\left(\lambda^{+}+\lambda^{-}\right)} e^{-\lambda|z|}+\frac{\tilde{B}_{z}^{0}}{s} e^{-k|z|} .
$$

Equation (3.11) shows that the transient behaviour of $B_{z}$ results from an induced field and the original steady state field. For large time, $\lambda^{+} \sim \lambda^{-} \sim k$, and so the field in (3.11) decays to zero, as required.

From the comments in the Introduction, there is particular interest in the time derivative of $B_{z}$, and from (3.11),

$$
\mathscr{F} L \partial_{t} B_{z}=-\frac{2 \tilde{B}_{z}^{0} k}{\lambda^{+}+\lambda^{-}} e^{-\lambda|z|}=\frac{2 \tilde{B}_{z}^{0} k\left(\lambda^{+}-\lambda^{-}\right)}{\mu \sigma s} e^{-\lambda|z|} .
$$


Equation (3.12) shows that $\partial_{t} B_{z}$ can be considered to result from two terms; that containing $\lambda^{+}$, and that from $-\lambda^{-}$. The former term is associated with transmission above the conductor, and the latter with transmission through the conductor. Consequently, the latter term is expected to be insignificant for early time.

Setting $\epsilon=0$, and taking the inverse Laplace transform [6] of (3.12) yields, for $z \geq 0$,

$$
\begin{aligned}
\mathscr{F} \partial_{t} B_{z} & =\frac{2 \tilde{B}_{z}^{0} k e^{-k z}}{\mu \sigma}\left[k \operatorname{erfc} \sqrt{\frac{k^{2} t}{\mu \sigma}}-\sqrt{\frac{\mu \sigma}{\pi t}} e^{-k^{2} t / \mu \sigma}\right] \\
& \simeq \frac{2}{\mu \sigma} \partial_{z}^{2} \mathscr{F} B_{z}^{0}+\frac{2}{\sqrt{\pi \mu \sigma t}}\left(\frac{\partial}{\partial z} \mathscr{F} B_{z}^{0}\right) e^{-k^{2} t / \mu \sigma},
\end{aligned}
$$

where the last approximation is true for small time. In (3.13), erfc is the error function [1]. However, for small values of time, the second term on the right hand side of (3.13) is negligible, since

$$
e^{-k^{2} t / \mu \sigma}=\mathscr{F}\left[\frac{\mu \sigma}{2 t} e^{-\mu \sigma\left(x^{2}+y^{2}\right) / 4 t}\right]
$$

and so for early times, the exponential factor dominates. Consequently, for $z \geq 0$, and for early times,

$$
\partial_{t} B_{z} \simeq \frac{2}{\mu \sigma} \partial_{z}^{2} B_{z}^{0}
$$

\section{The transient horizontal electric field}

The horizontal electric field results from two contributions: the decay of the initial field, and effects resulting from the image current source under the wires lying on the surface. These two effects are essentially independent, since current flow through the ground depends on the electrode positions, whereas the image current sources can take any path between the electrodes. Also, the initial electric field is independent of the position of the wires.

It is shown in the Appendix in (A.9) that the electric field above the conductor associated with the electrode currents (that is, the scalar potential, $\Psi$ ) reduces to zero in the quasi-steady approximation as soon as the current flowing through the wires is stopped. Thus the only contribution to the electric field above the conductor results from the vector potential, $A$. The aim in the remainder of this section is to give a physically motivated argument to emphasise the role of the surface wires in establishing the new horizontal electric fields on and above the conductor. A formal derivation of the same results is contained in the Appendix. 
It remains to evaluate the contribution to the horizontal electric field from the surface wires, for very small values of time, on and above the conducting surface. This is done by considering the contribution from each infinitesimal section of surface wire, and then summing all such terms.

Consider a right-handed Cartesian coordinate system, with unit normals $\left(e_{l}, e_{n}, e_{z}\right)$, where $e_{l}$ is directed in the direction of the infinitesimal section of surface wire. The unit vector $e_{z}$ is directed vertically upwards, and $e_{n}=e_{l} \times e_{x}$. From the relevant Maxwell equation,

$$
\partial_{l} E_{n}-\partial_{n} E_{l}+\partial_{t} B_{z}=0 .
$$

But from (3.14) for early time, on and above the surface $z=0$,

$$
\partial_{n} E_{l}=\frac{2}{\mu \sigma} \partial_{z}^{2} B_{z}^{0}
$$

since, from symmetry, $E_{n}=0$. From $\nabla \cdot B=0$,

$$
\partial_{z}^{2} B_{z}^{0}=-\partial_{z} \partial_{l} B_{l}^{0}-\partial_{z} \partial_{n} B_{n}^{0}=-\partial_{n} \partial_{z} B_{n}^{0}
$$

since, from the Biot-Savart Law (applicable to steady state fields), $B_{l}^{0}=0$. From (4.3) and (4.2),

$$
E_{l}=-\frac{2}{\mu \sigma} \partial_{z} B_{n}^{0},
$$

where the arbitrary function of integration has been set to zero, which is its value for unbounded values of $n$.

Applying the Biot-Savart Law to the infinitesimal length of wire,

$$
d B_{n}^{0}=\frac{\mu I}{4 \pi} \frac{\left(d l \times e_{r}\right) \cdot\left(e_{2} \times e_{l}\right)}{r^{2}}=\frac{\mu I|d l|}{4 \pi} \partial_{z}\left(\frac{1}{r}\right),
$$

where $r$ is the distance between the wire segment and the observation point, and $e_{r}$ is a unit vector directed from the wire segment to the observation point.

From (4.5) and (4.4),

$$
E=-\frac{2}{\mu \sigma} \partial_{z}^{2} \frac{\mu I d \mathbf{l}}{4 \pi r},
$$

showing that the early-time contribution to the horizontal electric field results from the initial steady state vector potential in (2.5). Consequently, from linearity, (2.5) and (4.6), the early time horizontal electric field satisfies

$$
E_{h}=-\frac{2}{\mu \sigma} \partial_{z}^{2} A_{h}^{0}
$$


where $A_{h}^{0}$ is the initial steady state vector potential from the surface wires (which are considered horizontal). This condition has also been derived more formally in the Appendix in (A.10).

For example, on the conducting surface, the early horizontal electric field generated by impulsively stopping the current in an infinitesimal vertical magnetic dipole is axial ((7) and (8) in [15]), and equals $3 I R_{0}^{2} /\left(2 \sigma R^{4}\right)$. This result follows from (2.6) and (4.7). The corresponding surface electric field from impulsively stopping the current in an infinitesimal horizontal electric dipole is $I d l /\left(2 \pi \sigma R^{3}\right)((7)$ and (13) in [13]), which follows from (4.7) and (2.5).

\section{Conclusion}

The main analytical result in this paper has been the derivation of the early time quasi-steady horizontal components of the electric field, on or above a uniformly conducting half-space, resulting from instantaneously interrupting initially steady electrical currents in surface wires. Equation (4.7) shows that this early time horizontal electric field results wholly from image currents established in the earth.

A similar analytical result was established in (3.14) for the early time behaviour of $\partial_{t} B_{z}$ on or above the conducting surface. However, the result in (3.14) follows from (4.7), because of one of Maxwell's equations. Both (3.14) and (4.7) agree with published results for the infinitesimal magnetic [9] and electric [13] dipole fields.

Perhaps the key result in this paper has been recognising that the conductivity value appearing in both (3.14) and (4.7) arises from the image currents, and so provides an indication of surface values of conductivity. In steady state methods, such as the Wenner array, the horizontal electric fields at the earth's surface resulting from the electrode currents are used. The horizontal electric field in (4.7) has zero contribution from the electrode currents, and arises instead wholly from the image currents under the wires. Consequently, different physical parameters are being sampled in steady state methods, and those using (4.7) and (3.14).

The magnetic field from an electrode is axial for both a uniform, and a horizontally layered, earth. Further, the inability of early field changes to significantly penetrate into the earth is also true for uniform or horizontally layered half-spaces. This suggests that (3.14) and (4.7) should remain valid for a horizontally layered earth, although this has not been proved in this paper.

Nevertheless, there are some (unrealistic?) situations in which extremely high conductivity lower layers may alter the apparent conductivity of surface layers. Consider a layer of thickness $z \mathrm{~m}$, conductivity $\sigma_{1} \mathrm{mho} / \mathrm{m}$, overlying a thick layer of conductivity $\sigma_{2} \mathrm{mho} / \mathrm{my}$. The time for fields to penetrate vertically through the upper layer is $t_{1} \sim \mu \sigma_{1} z^{2}$, while the time for fields to penetrate horizontally through the lower 
layer is $t_{2} \sim \mu \sigma_{2} r^{2}$. For lower transmission to affect the surface layers for early time requires $t_{2}<t_{1}$, or $r<\sqrt{\sigma_{1} / \sigma_{2}} z$. Since interest is in small values of $z$ and large values of $r$, this inequality will usually not be satisfied. Thus the early time quasi-steady fields will typically sample surface values of conductivity.

A key assumption in this paper was the quasi-steady approximation that $\epsilon=0$. This allowed the coefficients in (A.8) to be determined. This may reflect some indeterminacy in the mathematical system, since the same coefficients in (A.8) should result if the currents in the wires are stopped either impulsively, or over a time scale of the order of $\epsilon / \sigma$.

Several uses of (4.7) and (3.14) can be considered. For example, the majority of life on the solid earth depends on water relationships within the top metre or so of the earth. Electrical conductivity depends on water content. Consequently, (4.7) and (3.14) may yield information on the average state of water relations within the surface layers of the earth.

\section{Appendix}

Maxwell's equations can be written as

$$
\begin{aligned}
& B=\nabla \times A, \\
& E=-\partial_{t} A-\nabla \Psi,
\end{aligned}
$$

where $A$ and $\Psi$ are the vector and scalar potentials, respectively. Freedom in the choice of $A$ allows $A$ and $\Psi$ to be selected so that

$$
\nabla \cdot A+\mu \sigma \Psi+\mu \epsilon \partial_{t} \Psi=0
$$

which results in the wave equations

$$
\begin{aligned}
& \nabla^{2} A=\mu \sigma \partial_{t} A+\mu \epsilon \partial_{t}^{2} A, \\
& \nabla^{2} \Psi=\mu \sigma \partial_{t} \Psi+\mu \epsilon \partial_{t}^{2} \Psi-\rho / \epsilon,
\end{aligned}
$$

where $\rho$ is electrical charge density, assumed non-zero only on the surface of the conductor.

The boundary conditions are that $A_{x}, A_{y}$ and $\Psi$ are continuous at the boundary of the conductor. Charge conservation requires

$$
\nabla \cdot\left(\sigma E+\epsilon \partial_{t} E\right)=0 .
$$

The Fourier transform of the Laplace transform of components has the form

$$
\mathscr{F} L A=C^{ \pm} e^{-\lambda|z|}+\frac{\tilde{A}^{0}}{s} e^{-{ }^{k|z|}},
$$


where $C^{ \pm}$are position-independent coefficients.

Application of the boundary conditions then fixes

$$
\begin{aligned}
C_{x}^{+}=C_{x}^{-}, \quad C_{y}^{+} & =C_{y}^{-}, \\
\lambda^{+} C_{z}^{+}+\lambda^{-} C_{z}^{-} & =2 k \tilde{A}_{z}^{0} / s, \\
i k_{x}\left(C_{z}^{+}-C_{z}^{-}\right)-\left(\lambda^{+}+\lambda^{-}\right) C_{x} & =2 k \tilde{A}_{x}^{0} / s, \\
i k_{y}\left(C_{z}^{+}-C_{z}^{-}\right)-\left(\lambda^{+}+\lambda^{-}\right) C_{y} & =2 k \tilde{A}_{y}^{0} / s .
\end{aligned}
$$

These constraints are insufficient to fix the coefficients. The quasi-steady approximation is now made that $\epsilon=0$, and from (A.6), $E_{z}^{-}=0$ at the surface $z=0$. This leads to the quasi-steady conditions that

$$
\lambda^{+} C_{z}^{-}+\lambda^{-} C_{z}^{+}=-2 k \tilde{A}_{z}^{0} / s
$$

and so

$$
\begin{aligned}
C_{z}^{+} & =C_{z}^{-}=\frac{2 k\left(k-\lambda^{-}\right) \tilde{A}_{z}^{0}}{\mu \sigma s^{2}}, \\
C_{x} & =\frac{-2 k \tilde{A}_{x}^{0}}{s\left(k+\lambda^{-}\right)}, \quad C_{y}=\frac{-2 k \tilde{A}_{y}^{0}}{s\left(k+\lambda^{-}\right)} .
\end{aligned}
$$

The solution for $\Psi$ follows from (A.3) and (A.8). Above the conductor, and for the quasi-steady approximation,

$$
\mathscr{F} L \Psi^{+}=\frac{\left(k-\lambda^{-}\right)}{\mu \sigma s\left(k+\lambda^{-}\right)} \mathscr{F} \nabla \cdot A(0)=0
$$

since the initial vector potential, $A(0)$, is divergence-less above the conductor. The scalar potential therefore collapses to zero above the conductor in the quasi-steady approximation.

From (A.2), (A.7), (A.9) and the derivation leading to (3.14) for early time,

$$
\begin{aligned}
\mathscr{F} L E_{x}^{+} & =-s C_{x} e^{-k z}=\frac{2 k\left(\lambda^{-}-k\right) \tilde{A}_{x}^{0} e^{-k z}}{\mu \sigma s} \\
& \simeq-\frac{2 k^{2}}{\mu \sigma s} \tilde{A}_{x}^{0} e^{-k z}=-\frac{2}{\mu \sigma} \partial_{z}^{2} \mathscr{F} L A_{x}(0) .
\end{aligned}
$$

\section{References}

[1] M. Abramowitz and I. A. Stegun (eds.), Handbook of mathematical functions (Dover, U.S. Government Printing Office, Washington, DC, 1965). 
[2] A. Baños, Dipole radiation in the presence of a conducting half-space (Pergamon Press, Oxford, 1966).

[3] D. Corson and P. Lorrain, Introduction to electromagnetic fields and waves (W. H. Freeman, San Francisco, 1962).

[4] T. Lee, "The effect of displacement currents on time domain electromagnetic fields", Bull. Aust. Soc. Explor. Geophys. 12 (1981) 34-36.

[5] T. J. Lewis and T. J. Lee, "The detection of induced polarization with a transient electromagnetic system", IEEE Trans. Geosci. Remote Sensing 22 (1984) 69-80.

[6] G. E. Roberts and H. Kaufman, Table of Laplace transforms (W. B. Saunders, Philadelphia, 1966).

[7] J. A. Sommerfeld, Electromagnetic theory (McGraw-Hill, New York, 1949).

[8] J. A. Stratton, Electromagnetic theory (McGraw-Hill, New York, 1941).

[9] J. R. Wait, "The magnetic dipole over a horizontally stratified earth", Can. J. Phys. 29 (1951) 577-592.

[10] J. R. Wait, "Propagation of electromagnetic pulses in a homogeneous conducting earth", Appl. Sci. Res. B 8 (1960) 213-253.

[11] J. R. Wait, Geo-electromagnetism (Academic Press, New York, 1982).

[12] G. J. Weir, "Singular spin harmonics", J. Math. Phys. 20 (1979) 1648-1649.

[13] G. J. Weir, "Transient electromagnetic fields about an infinitesimally long grounded horizontal electric dipole on the surface of a uniform half-space", Geophys. J. R. Astr. Soc. 61 (1980) 41-56.

[14] G. J. Weir, "Electromagnetic multipole propagation in a homogeneous conducting whole space", J. Math. Phys. 23 (1982) 748-752.

[15] G. J. Weir, "Forerunners on conducting surfaces: the infinitesimal vertical magnetic dipole with displacement terms", Geophys. J. R. Astr. Soc. 81 (1985) 19-31. 